\title{
Energy Efficient Multipath Routing Protocol for WMSN's
}

\author{
A. V. Sutagundar*, S. S. Manvi**, Kirankumar. B. Balavalad*
}

\begin{abstract}
Routing in wireless Multimedia Sensor Networks (WMSN's) is a demanding task. This demand has led to a number of routing protocols which efficiently utilize the limited resources available at the sensor nodes. To increase the efficiency this paper proposes a protocol referred to be an Energy Efficient Multi-path Routing Protocol for WMSN's. It takes a view that, always using the minimum energy path deprives the nodes energy quickly and the time taken to determine an alternate path increases. It uses multiple paths between source and the sink which is intended to provide a reliable transmission environment with low energy consumption, by efficiently utilizing the energy availability of the nodes to identify multiple routes to the destination. The scheme is simulated for testing its operation effectiveness in terms of path setup time, data transmission time, node energy consumption time, and life time of sensor network.
\end{abstract}

Index Terms - WSN's, Routing protocols, Multipath Routing, Energy, Efficient Routing.

\section{INTRODUCTION}

Recent advancement in wireless communications and sensor technology have enabled the development of low-cost sensor networks. The sensor networks can be used for various application areas such as health, military, environmental monitoring, home, etc. Wireless sensor networks (WSN) have gained much attention recently. Usually a WSN consists of a large number of low-cost and low-energy sensors, which are deployed in the environment to collect observations and pre-process the observations. Each sensor node has its own communication capability to communicate with other sensor nodes or the central node (fusion center) via a wireless channel.

The position of sensor nodes need not be engineered or predetermined. This allows random deployment of nodes in inaccessible terrains or disaster relief operations. On the other hand, this means that sensor network protocols and algorithms must possess self-organizing capabilities. Another unique feature of sensor networks is the cooperative effort of sensor nodes. Sensor nodes are fitted with an onboard processor. Instead of sending the raw data to the

\footnotetext{
*Department of Electronics and Communication Engineering, Basaveshwar

Engineering College, Bagalkot-587102, INDIA, Email: ashok_ec@yahoo.com

$* * \bar{D}$ epartment of Electronics and Communication Engineering, REVA

Institute of Technology and Management, Bangalore-560064, INDIA, email:agentsun2002@yahoo.com

*Department of Electronics and Communication Engineering,

Basaveshwar Engineering College, Bagalkot-587102, INDIA, email: kiranb164@rediffmail.com
}

nodes responsible for the fusion, they use their processing abilities to locally carry out simple computations and transmit only the required and partially processed data

The challenges faced in designing sensor network systems and applications can be summarized as follows [1,2,3,4]: hardware constraints, bandwidth limitations, unreliable connectivity, real-time data logging, power efficient dynamic routing, self organization, decentralized information fusion, security, dynamic software architectures, and dynamic application specific executions.

Routing in sensor networks is very challenging due to several characteristics that distinguish them from contemporary routings: 1)It is not possible to build a global addressing scheme for deployment of sheer number of sensor nodes. Therefore classical IP-based protocols cannot be applied to sensor networks. 2) In contrary to typical communication networks almost all applications of sensor networks require the flow of sensed data from multiple regions to a particular sink. 3) Generated data traffic has significant redundancy in it since multiple sensors may generate same data within the vicinity of a phenomenon. Such redundancy needs to be exploited by the routing protocols to improve energy and bandwidth utilization. 4) Sensor nodes are tightly constrained in terms of transmission power, on-board energy, processing capacity, storage and thus require careful resource management.

The availability of low-cost hardware such as CMOS cameras and microphones has fostered the development of Wireless Multimedia Sensor Networks (WMSNs) [2], i.e., networks of wirelessly interconnected devices that are able to retrieve multimedia content such as video and audio streams, still images, and scalar sensor data from the environment ubiquitously. The concerns of routing in general differ significantly from the specialized service requirements of multimedia streaming applications [3].

All th existing protocols takes the minimum energy path. Where as the multi-path routing schemes distribute traffic among multiple paths instead of routing all the traffic along a single path. In multi-path routing it is necessary to know number of paths that are needed and choosing the appropriate paths in the total number of available paths [4]. Clearly, the number and the quality of the paths selected dictate the performance of a multipath routing scheme. The proposed work is intended to provide a reliable transmission of data for data synchronization at the destination on environment with low energy consumption. This is done by efficiently utilizing the energy availability and the received signal strength of the nodes to identify multiple routes to the destination.

The proposed protocol spreads the traffic over the nodes lying on different possible paths between the source and the 
sink in proportion to their residual energy and received signal strength. The rationale behind traffic spreading is by considering the energy so that the overall lifetime of the network will be increased. The sequence number is assigned to each packet of data for data synchronization at the destination. The objective is to assign more loads to under-utilized paths and less load to over-committed paths so that uniform resource utilization of all available paths can be ensured. We compare our proposed scheme with the directed diffusion and flooding protocols, Simulation results show that energy efficient adaptive multipath routing achieves better performance as compared to the traditional routing.

Some of the related works in multipath routing are as follows. Two Phase geographic Greedy Forwarding (TPGF) [7] routing algorithm has two phases, the first phase is responsible for exploring the possible routing path. The second phase is responsible for optimizing the explored routing path with the least number of hops. Multi-hop planer model transmits sensed data by forwarding to one of its neighbors which are closer to the sink node [8], [9], [10]. This approach uses data aggregation technique that enhances the efficiency of network by reducing the number of transmitting data.

Most of the multipath routing protocols are the extended versions of DSR [11] and AODV. Only a few research works adopt the geographical information to facilitate the on-demand disjoint multipath routing, one such routing protocol is Geography based Ad Hoc On demand Disjoint Multipath (GAODM) [12] in Ad Hoc networks. GAODM uses the push-reliable algorithm it focuses on how to use the push-reliable algorithm to find multiple node/edge disjoint paths based on the flow assignment. A node-Disjoint Parallel Multipath Routing algorithm (DPMR) [13] has two key problems, it relies on the algorithm proposed, and has the restrictions of using either clockwise region or anticlockwise region, which actually limits the number of routing paths.

Geographic Multicast Routing (GMR), a new multicast routing protocol for wireless sensor networks. It is a fully localized algorithm that efficiently delivers multicast data messages to multiple destinations. GMR optimizes the cost over progress ratio where the cost is equal to the number of neighbors selected for relaying and the progress is the overall reduction of the remaining distances to destinations. Maximally Radio-Disjoint Multipath Routing for Wireless Multimedia Sensor Networks [14] is basically based on path failure. This protocol maintains additional paths to serve as backup on primary path failure. Network Lifetime Maximization with Node Admission in Wireless Multimedia Sensor Networks [16]. Due to stringent QoS requirements its not possible to admit all the potential sensor nodes into the network, so this work addresses the node admission into the network, such that the network lifetime will be maximized.

The rest of the paper is organized as follows. Section 2 presents a proposed work. Section 3 depicts the simulation. Section 4 explains results and finally section 5 presents the conclusion.

\section{PROPOSED WORK}

Routing in wireless sensor networks is a demanding task. This demand has led to a number of routing protocols which efficiently utilize the limited resources available at the sensor nodes. All these protocols typically find the minimum energy path. In this paper we take a view that, always using the minimum energy path deprives the nodes energy quickly and the time taken to determine an alternate path increases. Multipath routing schemes distribute traffic among multiple paths instead of routing all the traffic along a single path. Two key questions that arise in Multipath routing are how many paths are needed and how to select these paths[4]. Clearly, the number and the quality of the paths selected dictate the performance of a Multipath routing scheme. We propose an energy efficient adaptive Multipath routing technique which utilizes multiple paths between source and the sink, adaptive because they have low routing overhead. This protocol is intended to provide a reliable transmission environment with low energy consumption, by efficiently utilizing the energy availability and the received signal strength of the nodes to identify multiple routes to the destination. Simulation results show that the energy efficient adaptive multipath routing scheme achieves much higher performance than the classical routing protocols, even in the presence of high node density and overcomes simultaneous packet forwarding.

In the proposed routing protocol the traffic is spread over the nodes lying on different possible paths between the source and the sink, in proportion to their residual energy and received signal strength. The rationale behind traffic spreading is that for a given total energy consumption in the network, at each moment, every node should have spent the same amount of energy. The objective is to assign more loads to under-utilized paths and less load to over-committed paths so that uniform resource utilization of all available paths can be ensured. Multipath routing is cost effective for heavy load scenario, while a single path routing scheme with a lower complexity may otherwise be more desirable.

\section{A. System Environment}

The sensor nodes are distributed randomly in the sensing field. A network composed of a sink node and many wireless sensor nodes in an interesting area is considered. The system environment is shown in figure 1. Assume that all nodes in the network are assigned with a unique ID and all nodes are participating in the network and forward the given data. The sensor nodes are assumed to be fixed for their lifetimes, and the identifier of sensor nodes is determined a priori. Additionally, these sensor nodes have limited processing power, storage and energy, while the sink nodes have powerful resources to perform any tasks or communicate with the sensor nodes. Once the nodes are deployed, they remain at their locations for sensing tasks. The sensor nodes can receive messages from other nodes. A node is selected to forward the data based on its available energy level and signal strength. Ideally, the greater the energy in the node and farther the node from the previous one, is the more likely to be selected as the next hop. The nodes which are not selected in this process are moved to the sleep mode in order to conserve power. The communication is assumed to be 
bidirectional and symmetric. The protocol replies with multiple routes from the source node to the sink quickly, and prepares the paths that efficiently balance the energy of the nodes. It also enables the selected nodes in the path to aggregate all the received packets during a short period of time and to transmit only the aggregated packet to the upstream node. Each node maintains a neighbor table for the routing protocol to function. The neighbor table contains an entry of all the selected neighboring nodes through which a node can transmit data..

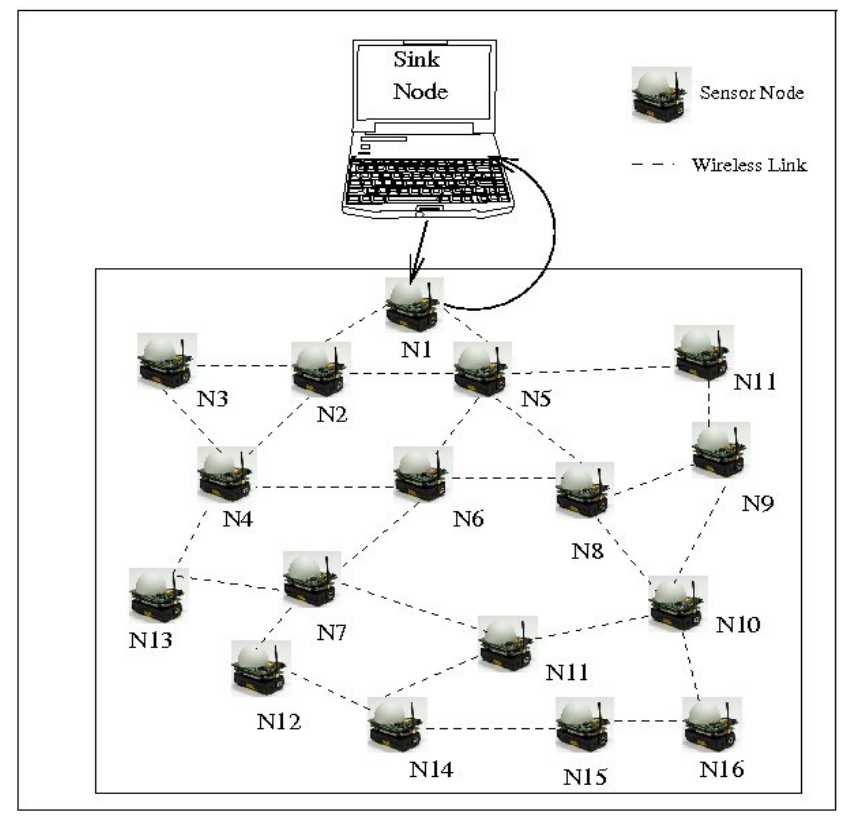

Fig. 1 System environment

\section{B. Multipath Routing}

This section describes the main components of multipath routing: path discovery, neighbor status table and the information routing algorithm.

1) Path Discovery: In path discovery phase sink diffuses the route request message to create neighboring nodes status table that is to maintain the addresses of all the nodes that are able to communicate the data with the source node. During this process route request message is exchanged between the sensor nodes. All the sensor nodes broadcast the route request message initially and maintain its own routing table. When the sensor node receives the information to be transmitted it transfers it to its neighboring nodes. Routes are established between the source and destination only when required hence it reduces the sensor node overhead. The multipath routing protocol computes the energy expense is less to transmit, receives and stores it in the routing table. Thereafter, it supports multipath information forwarding, not using the single path, so the energy consumption is distributed and the lifetime of network is prolonged.

Routing in general differ significantly from the specialized service requirements of multimedia streaming applications. Multiple routes in WMSNs are necessary to satisfy the desired data rate at the destination node. Benefits of selecting multiple paths among a WMSN: Reduced correlation among packet losses, Increase channel that can support the application's demands in QoS, the power consumption is more evenly spread in the network nodes extending longer life time, ability to adjust to arbitrary congestion occurrences in different parts of the network.

Routing in general differ significantly from the specialized service requirements of multimedia streaming applications. Multiple routes in WMSN's are necessary to satisfy the desired data rate at the destination node. Benefits of selecting multiple paths among a WMSN:1) Reduced correlation among packet losses, 2) increased channel rate that can support the applications demands in QoS, 3)the power consumption is more evenly spread in the network nodes extending longer life time, 4) ability to adjust to arbitrary congestion occurrences in different parts of the network. A sensor node is selected to forward the data, based on its available energy level and signal strength. Ideally, the greater the energy in the node and farther the node from the previous one, is the more likely to be selected as the next hop. The nodes which are not selected in this process are moved to the sleep mode in order to conserve power. The communication is assumed to be bidirectional and symmetric. The protocol replies with multiple routes from the source node to the sink

quickly, and prepares the paths that efficiently balance the energy of the nodes. It also enables the selected nodes in the path to aggregate all the received packets during a short period of time and to transmit only the aggregated packet to the upstream node. Each node maintains a neighbor table for the routing protocol to function. The neighbor table contains an entry of all the selected neighboring nodes through which a node can transmit data.

The network architecture is shown in the fig. 2. It consists of number of sensor nodes deployed randomly in a region with single base station. Here it is assumed that node $\mathrm{n} 20$ is the source and node $n 15$ is the destination. Nodes $n 1, n 2, n 3$, n6, n7, n8, n10, n11, n12, n13, n14, n15, n16, n17 and n18 are the intermediate nodes through which the different paths can be setup for the data transfer. Each node is limited by its transmission range which limits its capacity to communicate with all the remaining nodes directly. Hence it should use one or more intermediate nodes to communicate with the intended node. The nodes which lie within the transmission range of a node are said to be neighboring nodes for that particular node.

2) Information Routing: Once the multiple paths are discovered, source node transmits information packets with data rate initialized. Information packet comprises of information, sequence number, source id, and information length. Information packet is updated for every hop, which helps the sink node to monitor the conditions of the multiple routes being used. The initial data rate used for the routes may not be optimal for the duration of the connection.

Sink node redistributes the data rates over multiple path to optimize the usage of network resources periodically. In order to detect a path failure, the sink also monitors the inter-arrival delay of data packets on each path. When the delay is above a pre-determined threshold, the sink presumes that the path is broken. If the number of current working paths is equal to or lower than two, the sink will send a reset message to the source through the optimal path to indicate that sink starts to re-initiate the paths search phase. Otherwise, the sink readjusts the data rate allocation over other 
functional routes (i.e., sensor node uses different path every time to extend the lifetime of network system based on the information available in the routing table). This mechanism can avoid the path search phase being invoked frequently.

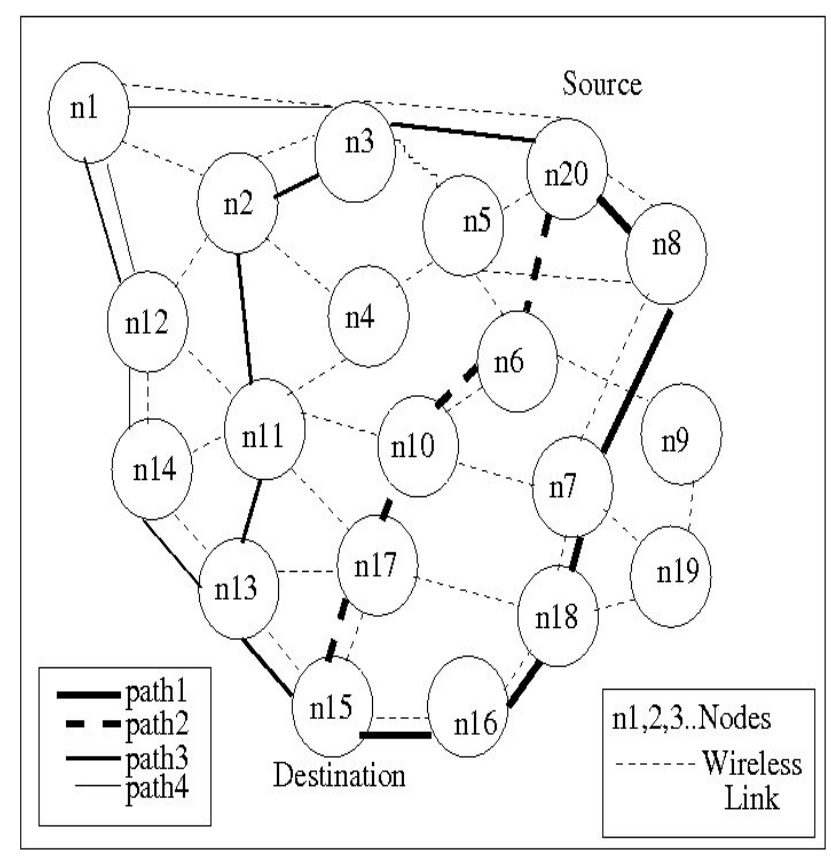

Fig. 2 Network architecture

\section{Algorithm}

Multipath routing algorithm followed by detailed functions used in the algorithm are as follows:

\section{Begin}

\section{Instances:}

Randomly generated data at each sensor node Operations:

1) Create neighboring table (): Create a neighboring node table

2) Search multiplepaths (): Search the multiple paths

3) Code Information (): Packet size and Code the information

4) Send Information (): Send the coded information

5) Reconstruct Decode (): Decode and synchronize the

reconstructed information

End

Create neighboring table ():

max nodes=number of nodes in the network, z [].x \&z [].y, $\mathrm{x}$ and $\mathrm{y}$ are co-ordinates of current node position, node $\mathrm{id}=$ node number, th $=$ threshold energy (th=5 unit), $z[]$.nodeenergy $=$ residual energy of node, node $u n=$ node which is within transmission range of current node, transmission range $=10$ meters

Begin

1) Get max nodes

2) Get the position of each node (z[].x,z[].y[])

3) Consider node with a node $\mathrm{id}=0$

4) Find the distance between node id and all other nodes

5) Dis tance $=\sqrt{\left((y 2-y 1)^{2}+(\infty 2-\infty 1)^{2}\right)}$

where $\mathrm{x} 1$ and $\mathrm{y} 1$ are position of current node, and $\mathrm{x} 2$ and $\mathrm{y} 2$ are position of the node from which the distance is to be calculated.

6) If (the distance<transmission range)

then

If(z[node un].nodeenergy $>$ Th)

Then update the neighbors of current node in neighbor table

7) Repeat the above steps for all other nodes

End

Get the number of sensor nodes and position of each node from the user. Initialize the first node with a value 0 , find distance between each of neighboring node by the distance formula. If the distance is within the transmission range and node energy is greater than the threshold energy then update the neighbors of current node in neighbor table.

\section{Search multiple paths ():}

Nomenclature:z[].nb[] = array to store the state of neighbors of each node, node id $=$ stores id of each node, route[] $=$ array to store the path, $z[] \cdot$ rout $[10]=$ structure to store all possible paths, $\mathrm{sr}=$ source, $\mathrm{d}=$ destination, $\mathrm{z}[]$.nodeenergy $=$ structure to store battery life. node.hop $=$ hop count , $z[] \cdot b=$ structure to store the status of each node( 1 $=$ sleep mode; $0=$ normal mode)

Begin

1) Get source and destination node id's from the user $($ Source $=s r$, destination $=d$ )

2) Get all information about each node and its neighbors from neighbor table $Z[i] \cdot n b[j]==1: j$ is neighbor of $I$

3) Check for destination if destination is neighbor of source then display as direct path from source to destination

4) From the source node start finding the route to destination ,first consider the neighbors of source node, check their status and then select the node with highest energy (node energy $==z[]$.nodeenergy).

5) Next make that node as source and continue the same process till the destination is reached.(Repeat step 4)

6) The nodes which are used in previous route are kept in sleep mode (status factor $==1: z[i] \cdot b==1$; which means that node $\mathrm{i}$ is in sleep mode) so that these should not be used in next path.

7) Again same procedure is applied to find the different possible paths from source to destination. and store the each path in a route array in the sequence as follows

8) Hop count: energy available : path from source to destination

9) Store all possible paths in a structure $z$ [].rout[]

10) End.

Get the source and destination node id's from the user and collects all information about each node and its neighbors from neighbor table. If destination is neighbor of source then display as direct path from source to destination. From the source node start searching the route to destination, first consider the neighbors of source node, check their status and then select the node with highest energy. Next make that node as source and continue the same process till the destination is reached. The nodes which are used in previous route are kept in sleep mode, the same procedure is used to find the different possible paths from source to destination and store the each path in a route array.

\section{Code Information ():}


Nomenclature: inter data [ ] = to store inter mediate data, $\mathrm{z}[\mathrm{sr}]$.nodevalue $=$ data generated at the source, $\mathrm{sr}=$ source node, data $=$ variable used to store the intermediate data.

Begin

1) Get the data generated at the source node (user input for simulation) Data $=\mathrm{z}[\mathrm{sr}]$.nodevalue

2) Unpack the data

3) convert unpacked data to binary (nibble)

4) Assign sequence number and calculate the checksum for each nibble

5) Arrange data in the data format shown below which forms a packet (8-bit) Sequence no (2 bits) Data(4 bits) checksum(2 bit)

6) Store these data packets in array inter data[ ]

7) Now data is ready for transmission

End

\section{send Information():}

Nomenclature: $z[]$.nodeenergy $=$ the residual energy of node, no of paths $=$ the number of possible paths, threshold $=$ 5 unit, inter data[] $=$ intermediate data[].nodevalue $=$ data at the node, nod $=$ variable to store the node $\mathrm{id}$, node $\mathrm{id}=$ node number.

Begin

1) Get the intermediate data

2) Check for path failure

3) If any path is failed due to insufficient energy of intermediate node or due to malfunctioning of node then delete the corresponding path (Insufficient energy less than threshold)

4) If (no of paths $>=$ the number of data packets) Then send one packet through each path

5) If (no of paths $<$ the number of data packets) Then send one packet through each path and remaining packets through highest energy path

6) Get the node id's of intermediate nodes of current path Transfer of data from one node to another node (nod $=\mathrm{Z}$ [].rout[] z [nod+1].nodevalue $=$ Z[nod].nodevalue)

7) After one transmission reduce the battery life by 5 units $z[i]$.nodeenergy $=z[i]$.nodeenergy -5

8) Repeat the steps 6 and 7 till the data reaches the destination

9) End.

Get the intermediate data and check for path failure, if any path is failed due to insufficient energy of intermediate node or due to malfunctioning of node then delete the corresponding path. If number of paths are greater than the number of data packets, then send one packet through each path. If number of paths is less than the number of data packets, then send one packet through each path and remaining packets through highest energy path. Get the node id's of intermediate nodes of current path, then transfer data from one node to another node. After one transmission reduce the battery life by 5 units.

\section{Reconstruct Decode ():}

Nomenclature: seq no $=$ sequence number of data packet used for synchronization, $z[]$.nodevalue $=$ data at the node $\mathrm{d}$ (destination), $\mathrm{d}=$ destination id.

Begin

1) Get the data from the destination node arrived through different paths Final data $=\mathrm{z}[\mathrm{d}]$.nodevalue

2) Decode the final data Data format Sequence no (2 bits) actual data(4 bits) checksum(2 bit)

3) Extract the actual data from the coded data and arrange the data packets according to their seq no

4) Store the actual data

End

Collect the data from destination node arrived through different paths. After collecting data from the destination node, extract the actual data from the coded data and arrange the data packets according to their sequence number. Store and display the actual data.

\section{Simulation Model And Performance Metrics}

We have carried out the simulation of the Multipath Routing Protocol for WMSN's. The proposed model has been simulated in various network scenarios on a Pentium-4 machine by using $\mathrm{C}$ tool for the performance and effectiveness of the approach. In this section of the paper simulation model, simulation procedures are discussed.

\section{A. Simulation Model}

Proposed scheme is simulated in various network scenarios to access the performance and effective of an approach. The proposed simulation model considers $\mathrm{N}$ number of nodes in the area of length $L$ and breadth B. Each node transmits a maximum of $\mathrm{P}$ packets at various times during simulation and their transmission range is $\mathrm{R}$ sq meters. The work considers parameters such as battery life of each node which is varied over an arbitrary range of values. Assuming that, initially there are no malicious nodes and all nodes have random amount of battery charge, We first detect whether the source, destination and intermediate nodes have sufficient battery capacity for communication. Depending upon the battery life and range of transmission we find out the neighbors of each node and store them in a neighbor table which is to be updated dynamically. With the help of this neighbor table we find out different routes from source to destination. If suppose one node is used in one route then it should not be used in other route to avoid collision of data while transmission. To achieve this we use a status factor which indicates the status of each node.

Routes are generated using proposed routing protocol based on the signal strength Th, energy status $\mathrm{Ei}$, and distance between the neighboring nodes $\mathrm{Di}$. The propagation delay of each link is measured as Pd. In simulation free space propagation model is used with propagation constant .

\section{B. Simulation Inputs}

To illustrate some results of the simulation, we have taken $\mathrm{L}=100, \mathrm{~B}=50 \mathrm{sq}$ meters and $\mathrm{N}=10$ to $100, \mathrm{R}=10$ meters, rate change $\operatorname{pos}=0$, energydroppertransmission $(8-$ bit $)=5$ milliwatts, $\mathrm{Th}=(50 \%, 60 \%, 70 \%)$, communication radius $\mathrm{r}=10 \mathrm{mts}, \mathrm{D}=(5,10,15)$ meters, Propagation Constant _ $=3.5$. Each of the simulation executes in varying seconds.

Begin

- Generate the WSN for the given area and number nodes.

- Apply the proposed multipath routing protocol.

- Compute the performance of the system. 
End

\section{Performance Parameters}

(1) Node energy consumption: The node energy consumption measures the average energy dissipated by a node in order to transmit the data packet from source to destination.

(2) Network Life Time: It is total time during which the network is alive. As the sensor node energy depletes life time of the network is reduced.

(3) Path setup time: This is the time required to find the number of paths from source to destination

Time required to set up the path $=($ time required to find the highest energy node + time required to store the paths)

\section{RESULTS}

In this section, as a measure of performance we analyze the simulation results of proposed scheme. The result presented demonstrates the effectiveness of proposed work. Simulations are repeated for several times to evaluate the performance of the system.

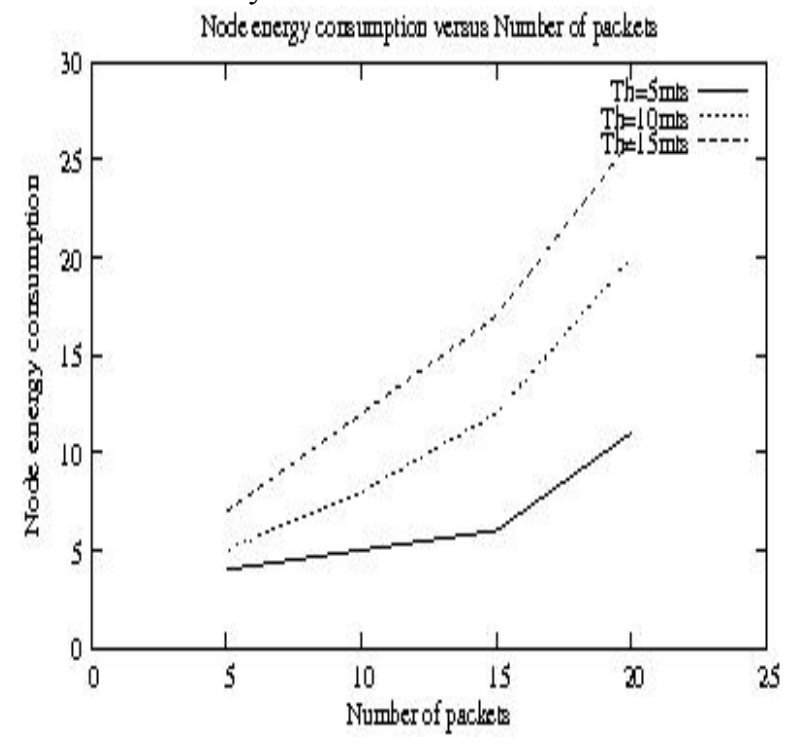

Fig. 3. Node energy consumption versus Number of packets

Figure 3 shows that Node energy consumption Versus Number of packets sent. From the figure we can depict that as number of packet sent increases the amount of energy consumed will also increases. As the threshold signal strength (transmission range) the number of nodes in the route also increases. Therefore as the signal strength increases the energy consumed to transmit the data also increases.

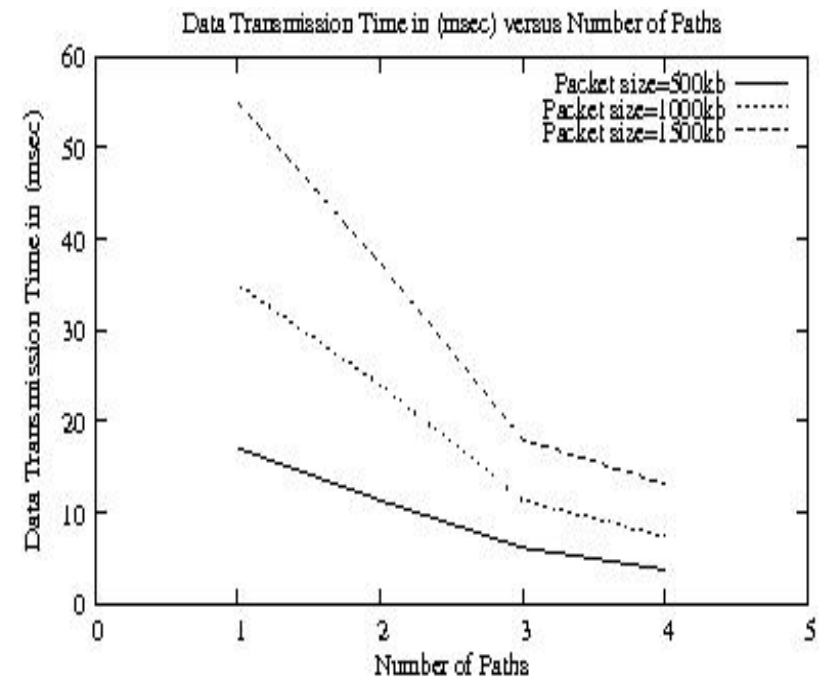

Fig. 4. Data Transmission Time (msec) versus Number of Paths

Figure 4 shows the Data transmission time Versus the Number of paths with varying data size. The packets are sent simultaneously sent over the multiple paths, therefore as there is increase in the number of paths the amount of time required to transmit the data is also decreased. The simulations are carried for varying data size. Path setup time versus number of nodes

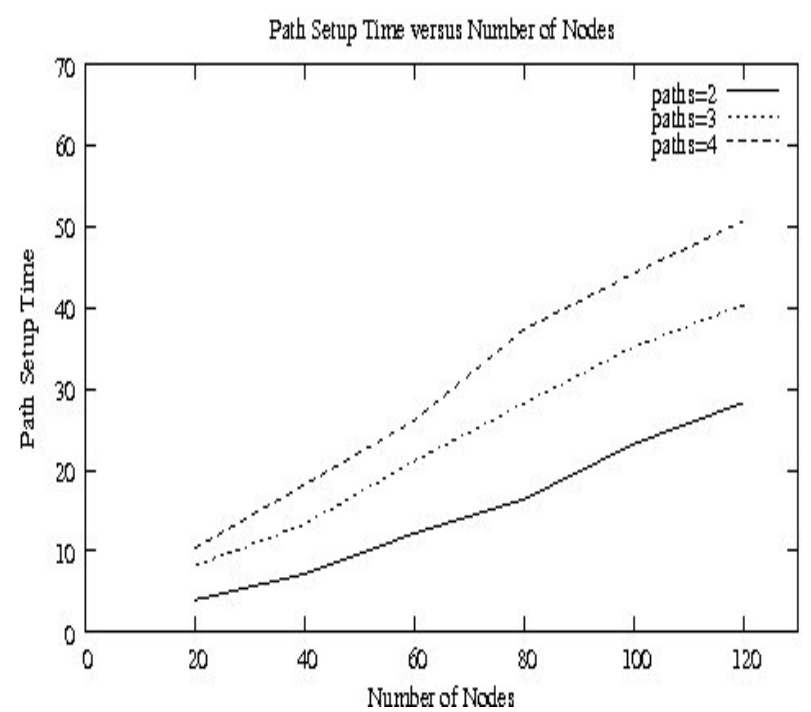

Fig. 5. Path Setup Time versus Number of Nodes

Figure 5 shows that as Path setup time versus number of nodes. With the increase in the number of nodes of nodes path setup time also increases because the number of sensor nodes in path also increases. Path setup time includes the neighboring node updating time and distance calculation between the sensor nodes. The required to calculate the distance between the neighboring nodes is given by

$$
\mathrm{TD}=\mathrm{Td} \times(\mathrm{N}-1)
$$

where $\mathrm{TD}=$ time required to calculate the distance of each node from all other nodes, $\mathrm{N}=$ number of nodes, $\mathrm{Td}=$ time required to calculate distance between any two nodes.

Total Time $(\mathrm{T})=[\mathrm{T} \mathrm{d}+\mathrm{S} \times \mathrm{T} \mathrm{n}] \times \mathrm{N}$

where $\mathrm{Tn}=$ time required to update a neighbor, and $\mathrm{S}=$ Average number of neighbors of all the nodes. 
Figure 6 shows the Life time of the sensor network versus the sensor node density. As the number of sensor node increases the more number of sensor involves in the communication hence more network energy is depletes. From the figure 6 we can notice that as there is increase in the number of nodes in unit area network life time decreases.

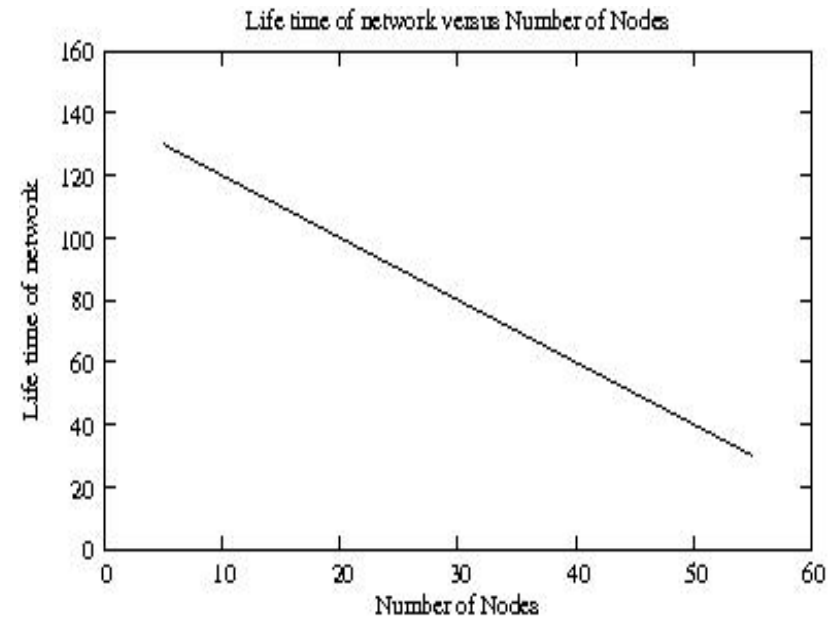

Fig. 6. Life Time of Network versus Node of Nodes

Figure 7 presents path setup time versus number of nodes with varying threshold transmission range. As the number of nodes increases there is increase in the setup time. With increase in transmission range more time is needed in order to setup the time.

Path Setup Time versus Number of Nodes

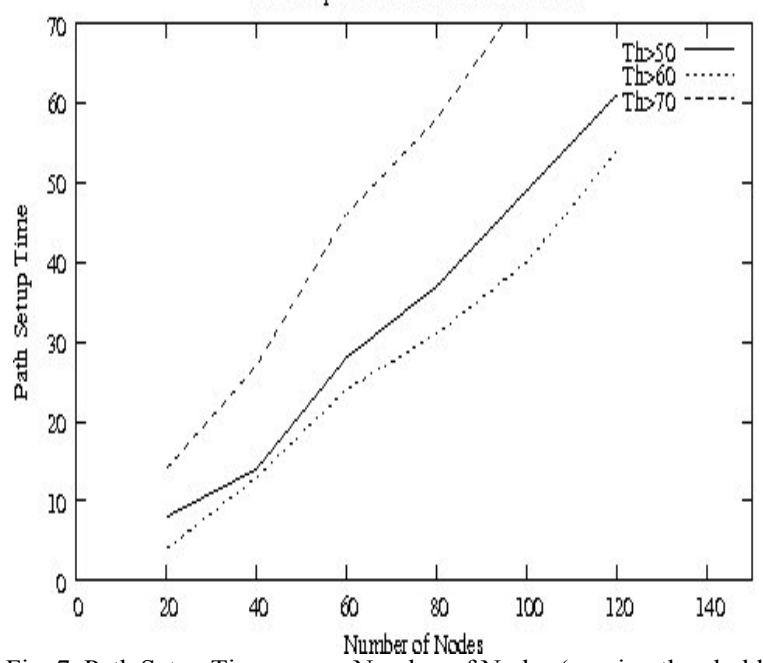

Fig. 7. Path Setup Time versus Number of Nodes (varying threshold transmission range

\section{CONCLUSION}

The main factors to be taken into consideration in case of WSN are battery life and fault tolerance. Since each node is minute and has less battery capability. We have to proposed energy efficient protocol for WMSN's which is capable of searching multiple paths and aims to allocate the traffic on each path optimally. Distributing the load to the nodes significantly impacts the system lifetime. Simulation results show that proposed scheme has higher node energy efficiency. It also provides data synchronization and it has fault tolerance capability.

\section{REFERENCES}

[1] W.M.Merrill, K.Sohrabi, "Open Standard Development Platforms for is0ributed Sensor Networks", Proc. SPIE, Unattended Ground Sensor technologies and Applications Iv (AeroSense 2002), Vol. 4743, pp. 327-337, Orlando, FL, April 2002.

[2] P.K. Willett, P.F. Swaszek, and R.S. Blum, "The good, bad, and ugly:distributed detection of a known signal in dependent Gaussian noise", IEEE Trans. Signal Processing, Vol. no.48, pp. 32663279, Dec. 2000.

[3] E. Drakopoulos and C.C. Lee, "Optimum multisensor fusion of correlated local decisions", IEEE Trans. on Aerospace and Elect. Syst., Vol.27. no 4, pp.593605, July 1991.

[4] Chen T. M, Luo R.C., "Multilevel Multiagent Based Team Decision Fusion for Autonomous Tracking System", Machine Intelligence Robotic Control, Vol 1, no.2, pp. 63-69,1999.

[5] I.F.Akyildiz, W.Su, Y. Sankarasubramaniam, E. Cayirci, "Wireless Sensor Networks: a survey", Computer Networks, The International Journal of Computer and Telecommunication Networking,vol. 3, No. 8, pp.393-422, ISSN: 1389-1286, 2002.

[6] I. Akyildiz, T. Melodia, and K. R. Chowdhury, "A survey of wireless multimedia sensor networks", Computer Networks, Vol.51: pp. 921960, 2007.

[7] Akkaya K., M. Younis, "A survey of routing protocols in wireless sensor networks",Ad Hoc Network, Elsevier, Vol.3, No. 3, pp.325349, 2005.

[8] Jing Zhou and David De Roure, "Designing Energy-Aware Adaptive Routing for Wireless Sensor Networks", The 6th International Conference on ITS Telecommunications, 21-23, June2006, Chengdu, China.

[9] Ying-Hong Wang, Chih-Hsiao Tsai, Hung-Jen Mao, "HMRP: Hierarchy-Based Multipath Routing Protocol forWireless Sensor Networks", Journal of Science and Engineering, Vol. 9, No 3, pp. 255-264, 2006.

[10] Yuwang Yang, Chunshan Zhong, Yamin Sun , Jingyu Yang, ”Energy Efficient Reliable Multi-path Routing Using Network Coding for Sensor Network", IJCSNS International Journal of Computer Science and Network Security, Vol.8, No.12, December 2008

[11] Y Chen, E Chan and Song Han, "Energy efficient multipath routing in large scale sensor networks with multiple sink nodes", The $6^{\text {th }}$ International Workshop on Advanced Parallel Processing Techniques, pp.390399, Hong Kong, 2005.

[12] Deepak Ganesan, Ramesh Govindan, Scott Shenker, Deborah Estrin, "Highly-Resilient, Energy-Effcient Multipath Routing in Wireless Sensor", Networks Mobile computing and Communications Review, Vol.1, No. 2.

[13] Sonia Waharte and Raouf Boutaba, "Totally Disjoint Multipath Routing in Multihop Wireless Networks", IEEE International Conference on Communications, ICC '06, Vol. 12 , pp. 5576-5581, 2006

[14] Zijian Wang, Eyuphan Bulut, and Boleslaw K. Szymanski ,’Energy Efficient Collision Aware Multipath Routing for Wireless Sensor Networks", Proc. International conference on Communication, ICC09,Dresden, Germany, June, 2009

[15] Moufida Maimour, "Maximally Radio-Disjoint Multipath Routing for Wireless Multimedia Sensor Networks", Proc. of the 4th ACM workshop on Wireless multimedia networking and performance modeling, pp.26-31, Vancouver, British Cloumbia, Canada, 2008.

[16] Francisco Rocha, Antnio Grilo, and Paulo Rogrio Pereira B. Marchi, A. Grilo, and M. Nunes, "DTSN Distributed Transport for Sensor Networks,In Proc. of the IEEE Symposium on Computers and Communications (ISCC07), Aveiro, Portugal, 2007.

[17] Khoa T. Phan, Rongfei Fan, Hai Jiang,"Network Lifetime Maximization with Node Admission in Wireless Multimedia Sensor Networks", IEEE Transactions on vehicular technology, vol.58, No.7, September 2009.

A.V.Sutagundar completed his M. Tech from Visvesvaraya Technological University Belgaum, Karnataka.He is pursing his $\mathrm{PhD}$ in the area of Content Based Information Retrieval in wireless Networks using Mobile Agents. Presently he is serving as a Assistant Profesor, Department of Electronics and Communication Engineering, Basaveshwar Engineering College, Bagalkot, Karnataka. His areas of interest include Signal and system, Digital Signal Processing, Digital Image Processing, Multimedia Networks, Computer communication networks, Wireless networks, Mobile ad-hoc networks, Agent technology. He has published 10 papers in referred National/International Conferences. 
S. S. MANVI completed his $\mathrm{PhD}$ from Indian Institute of Science, Bangalore. Presently he is serving as a Professor of Electronics and Communication Engineering, REVA Institute of Technology and Management, Bangalore. His areas of interest include wireless/wired network, AI applications in network management, E-commerce, Grid Computing and Multimedia Communications. $\mathrm{He}$ has published over 25 papers in referred National/International Journals and 60 papers in referred National/ International Conferences. He has coauthored books Communication Protocol Engineering and Computer Concepts and C Programming published by PHI. He is a reviewer of several national/international journals. $\mathrm{He}$ is a member of IEEE USA, Fellow of IETE, India Fellow of IE, India and member of ISTE, India. He has been included in Marquis Whos who in world and International Biographies of Cambridge, London in the year 2006.

Kirankumar B. Balavalad completed his B.E. in Electronics and Communication Engineering from Visvesvaraya Technological University Belgaum, Karnataka. He is pursuing his M.Tech. in Digital Communications. His areas of interest include Computer communication networks, Wireless networks, Digital communications and Multimedia communications. 